\title{
Variation in Effective Stimulus Patterns for Induction of Long-Term Potentiation Across Different Layers of Rat Entorhinal Cortex
}

\author{
Sung Hwan Yun, ${ }^{1}$ Inhee Mook-Jung, ${ }^{2}$ and Min Whan Jung ${ }^{1,2}$ \\ ${ }^{1}$ Neuroscience Laboratory, Institute for Medical Sciences and ${ }^{2 B}$ Brain Disease Research Center, Ajou University School of \\ Medicine, Suwon 442-721, Korea
}

\begin{abstract}
Neuronal activities in superficial (II and III) and deep (V and VI) layers of the entorhinal cortex (EC) are preferentially modulated by theta and sharp wave (SPW) EEG, respectively. We investigated the possibility that distinct EEG patterns represent optimal stimulus patterns for induction of long-term potentiation (LTP) in different layers of the EC. We examined effects of three different stimulation patterns on LTP induction in layers I, II-III, and $\mathrm{V}$ of medial $\mathrm{EC}$ slices of the rat. The stimulation patterns we used were a single, long high-frequency train (1 sec at $100 \mathrm{~Hz}$, repeated $3 \times$ ), theta burst stimulation [TBS; 10 bursts (four pulses, $100 \mathrm{~Hz}$ ) at $5 \mathrm{~Hz}$, repeated $3 \times$ ], and SPW-like burst stimulation [ SPWBS; three bursts (20 pulses, $200 \mathrm{~Hz}$ ) at $2 \mathrm{~Hz}$,
\end{abstract}

Knowledge about hippocampal interactions with other cortical sites is crucial for understanding the neural mechanisms by which the brain organizes memories. One of the most important brain structures for the study of hippocampal-neocortical interactions is the entorhinal cortex (EC), which mediates the majority of reciprocal connections between the hippocampus and the neocortex. The EC shows both areal and laminar differentiation in its connection with the hippocampus and neocortex. Anatomical segregation is observed along the mediolateral axis of the EC in its afferent, efferent, and associational projections, suggesting that different rostrocaudal strips of the EC may operate independently in processing neocortex- and hippocampus-originated information (Witter, 1986, 1993; Swanson et al., 1987; Dolorfo and Amaral, 1998). Regarding laminar differentiation, EC superficial layers (I-III) receive projections from unimodal and polymodal association areas via the perirhinal cortex and then provide the majority of input projections to the hippocampus, whereas hippocampal output projections terminate mostly in EC deep layers (IV-VI), which send extensive projections back to the neocortex (Swanson et al., 1987; Witter, 1993). Intrinsic associational fibers originating from superficial layers are confined within the same layers, whereas those from deep layers project to deep as well as superficial layers of the EC (Dolorfo and Amaral, 1998). This organizational scheme suggests that the superficial and deep EC

\footnotetext{
Received Sept. 19, 2001; revised Dec. 5, 2001; accepted Dec. 18, 2001.

This work was supported by the Neurobiology Research Program from the Korea Ministry of Science and Technology, the Korea Science and Engineering Foundation (KOSEF) grant through the Brain Disease Research Center at Ajou University, and the KOSEF project number R01-1999-00021. We thank Loren Frank and William Skaggs for commenting on an earlier version of this manuscript.

Correspondence should be addressed to Min Whan Jung, Neuroscience Laboratory, Institute for Medical Sciences, Ajou University School of Medicine, Suwon 442-721, Korea. E-mail: min@madang.ajou.ac.kr.

Copyright (ㄷ) 2002 Society for Neuroscience $\quad 0270-6474 / 02 / 220001-05 \$ 15.00 / 0$
}

repeated $6 \times$ ]. Similar degrees of LTP were induced by the three stimulation patterns in layer I. In layers II-III and layer V, however, the largest degrees of LTP were induced by TBS and SPWBS, respectively. These results suggest that burst stimulation constructed to mimic naturally occurring patterns of neuronal activity in the corresponding layer is optimal for LTP induction in layers II-III and layer V of the EC. The differences may play important roles in shaping hippocampal-neocortical interactions in encoding and retrieval of memory.

Key words: long-term potentiation; theta; sharp wave; hippocampus; entorhinal cortex; memory

mainly function as input and output structures of the hippocampus, respectively.

Physiological studies also suggest functional segregation between the superficial and deep EC. Theta frequency EEG dominates the hippocampal formation when an animal is actively exploring the surrounding environment, and sharp waves (SPWs) are observed during such behavioral states as awake immobility, consummatory behavior, and slow wave sleep (Buzsaki et al., 1983). Superficial EC neurons are phase-related to theta oscillation, but surprisingly indifferent to SPW-associated population bursting occurring within deep layers. In contrast, deep EC neurons fire in synchrony with SPWs (Chrobak and Buzsaki, 1994). Regarding theta modulation of deep EC neurons, they are either indifferent to theta EEG (Chrobak and Buzsaki, 1994) or only putative excitatory neurons, but not all fast-spiking neurons are theta modulated (Frank et al., 2001). These results indicate differential modulation of superficial and deep EC neurons by theta EEG and SPWs. Moreover, coding properties differ in interesting ways between superficial and deep EC neurons (Frank et al., 2000), suggesting different roles played by the superficial and deep EC.

These findings suggest that characteristics of long-term synaptic plasticity underlying mnemonic operations of the EC may be

This article is published in The Journal of Neuroscience, Rapid Communications Section, which publishes brief, peerreviewed papers online, not in print. Rapid Communications are posted online approximately one month earlier than they would appear if printed. They are listed in the Table of Contents of the next open issue of JNeurosci. Cite this article as: JNeurosci, 2002, 0:RC214 (1-5). The publication date is the date of posting online at www.jneurosci.org.

http://www.jneurosci.org/cgi/content/full/6148 


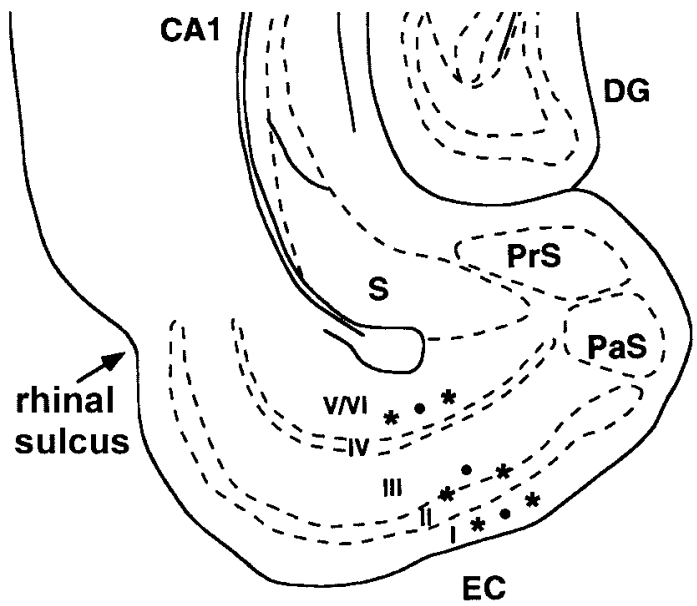

Figure 1. Placement of stimulating and recording electrodes. Horizontally sectioned medial EC slice preparations were used throughout the study. A recording electrode $(\bullet)$ was placed in layer I, superficial layer III, or layer $\mathrm{V}$ of the medial EC in response to electrical stimulation of layers I, II-III, or V, respectively, by two stimulating electrodes (*). EC, Entorhinal cortex; $P r S$, presubiculum; $P a S$, parasubiculum; $S$, subiculum; $D G$, dentate gyrus. The figure has been adapted from Amaral and Witter (1995), with permission from the publisher.

distinguished in different layers of the EC. Previous studies have shown that different layers of the EC support long-term potentiation (LTP) (Alonso et al., 1990; Yun et al., 2000a; Cheong et al., 2001). In these studies theta burst stimulation (TBS), which was patterned after hippocampal theta EEG, was used for LTP induction. Because superficial and deep EC neurons are preferentially modulated by distinct patterns of EEG (theta and SPWs, respectively), there exists a possibility that optimal induction of LTP is achieved by different patterns of stimulation in different layers. In the hippocampus, optimal stimulus patterns for inducing LTP are different across different regions (Yeckel and Berger, 1998). The primary objective of the present study was to test this possibility in the EC. The results indicate that optimal stimulus patterns for LTP induction are indeed different across different layers of the EC, and that they are related to naturally occurring patterns of neuronal activity.

\section{MATERIALS AND METHODS}

Horizontally sectioned $400-\mu \mathrm{m}$-thick entorhinal cortical slices were prepared from 150-200 gm male Sprague Dawley rats as previously described (Yun et al., 2000b). Animals were decapitated after anesthesia with halothane, and the brain was quickly removed and placed in cold artificial CSF (ACSF). The experimental protocol was approved by the Ethics Review Committees for Animal Experimentation of Ajou University School of Medicine. The ACSF contained (in mM): $\mathrm{NaCl} 124$, $\mathrm{KCl} 3, \mathrm{KH}_{2} \mathrm{PO}_{4}$ 1.2, $\mathrm{CaCl}_{2} 3.4, \mathrm{MgSO}_{4} 2.5, \mathrm{NaHCO}_{3} 26$, D-glucose 10, and L-ascorbate. Slices were transferred to an interface chamber that was constantly perfused with oxygenated ACSF $\left(95 \% \mathrm{O}_{2}\right.$ and $\left.5 \% \mathrm{CO}_{2}\right)$ and maintained at $35 \pm 1{ }^{\circ} \mathrm{C}$. Slices were allowed to recover for $\sim 1 \mathrm{hr}$, and then monosynaptic field potentials were recorded in layers I, superficial layer III, and V of the medial EC in response to electrical stimulation of layers I, II-III, and V, respectively (Fig. 1). Two stimulating electrodes were used in each case. The stimulating electrodes were constructed of twisted strands of stainless steel wires (113 $\mu \mathrm{m}$ outer diameter), and the recording electrodes were glass micropipettes filled with $2 \mathrm{M} \mathrm{NaCl}$ (1-5 $\mathrm{M} \Omega)$. Stimulation pulses $(0.1 \mathrm{msec})$ were delivered every $20 \mathrm{sec}$ at currents ranging from 50 to $450 \mu \mathrm{A}$ (layer I: $150-450 \mu \mathrm{A}$; layers II-III and $\mathrm{V}: 50-150 \mu \mathrm{A})$ to obtain baseline responses $(10-20 \mathrm{~min})$ that were approximately half-maximal.

LTP was induced by applying long high-frequency train (LHFT), TBS, or SPW-like burst stimulation (SPWBS). LHFT was a long highfrequency stimulus train $(1 \mathrm{sec}$ at $100 \mathrm{~Hz})$. Three episodes of LHFT were repeated at $10 \mathrm{sec}$ intervals. TBS consisted of 10 high-frequency bursts (four pulses at $100 \mathrm{~Hz}$ ) repeated at $5 \mathrm{~Hz}$ (Larson et al., 1986). Three episodes of TBS were applied at $10 \mathrm{sec}$ intervals. SPWBS consisted of three high-frequency bursts $(20$ pulses at $200 \mathrm{~Hz})$ repeated at 2 Hz. Six episodes of SPWBS were applied at $5 \mathrm{sec}$ intervals. During tetanic stimulation, stimulus duration was increased to a level that induced maximal field potential responses. Responses were amplified $100 \times$, filtered at bandpass $1 \mathrm{~Hz}-3 \mathrm{kHz}$, and digitized by a personal computer at $10 \mathrm{kHz}$. The program NAC (Eclectek, Irvine, CA) was used for collection and analysis of data. Evoked field potential responses were assessed by measuring the initial slope. The magnitude of LTP was assessed by measuring percentage of increase of averaged responses over baseline at 20-30 min after tetanic stimulation. A one-way ANOVA followed by post hoc least significant difference (LSD) test was used to compare the magnitudes of LTP induced by the three types of stimulation in the different layers of the EC. A $p$ value $<0.05$ was used as the criterion for a significant statistical difference. Results are expressed as mean \pm SEM.

\section{RESULTS}

\section{LTP induction in layer I}

The amplitude of the evoked field potential was small (maximum amplitude: $1 \sim 1.5 \mathrm{mV}$ ), and it was difficult to acquire stable baseline responses in layer I. When a stable baseline was obtained, however, LTP was reliably induced by LHFT, TBS, and SPW BS (Figs. $2 A, 3$ ). The magnitudes of LTP induced by LHFT, TBS, and SPWBS were $20.5 \pm 7.7 \%(n=10), 24.8 \pm 7.2 \%(n=$ $10)$, and $26.9 \pm 5.7 \%(n=10)$, respectively (Fig. 3$)$. The average magnitudes of LTP induced by the three types of stimulation were not significantly different in layer I (one-way ANOVA; $p>$ $0.05)$.

\section{LTP induction in layers II-III}

The amplitude of the evoked field potential was largest in layers II-III (maximum amplitude, $\sim 5 \mathrm{mV}$ ). The magnitudes of induced LTP were $13.4 \pm 4.1 \%(n=11), 33.1 \pm 5.4 \%(n=10)$, and $24.3 \pm 4.4 \%(n=10)$ by LHFT, TBS, and SPWBS, respectively (Figs. $2 B, 3$ ). The three stimulation patterns had significantly different effects on the magnitude of LTP (one-way ANOVA; $p<$ 0.05). A post hoc test (LSD) indicated a significant difference between TBS and LHFT $(p<0.01)$ (Fig. 3).

\section{LTP induction in layer $\mathbf{V}$}

In layer $\mathrm{V}$ the maximum amplitude of the field potential was $\sim 3$ $\mathrm{mV}$. The average magnitudes of LTP induced by LHFT, TBS, and SPWBS were $11.4 \pm 5.6 \%(n=16), 16.1 \pm 5.6 \%(n=19)$, and $33.8 \pm 4.9 \%(n=16)$, respectively (Figs. $2 C, 3)$. The three stimulation patterns had significantly different effect on the magnitude of LTP (one-way ANOVA; $p<0.05$ ). A post hoc test (LSD) indicated significant differences between LHFT and SPWBS $(p<0.01)$ and between TBS and SPWBS $(p<0.05)$ (Fig. 3).

\section{DISCUSSION}

Input and output projections of the hippocampus are primarily segregated in the EC (see introductory remarks). This organization provides an opportunity for separately investigating input and output pathways of the hippocampus. Previous studies in this laboratory, investigating characteristics of synaptic plasticity in superficial and deep layers of the EC, were unable to find clear differences in characteristics of LTP between layers II-III and V (Yun et al., 2000a,b; Cheong et al., 2001). In the present study we examined the effectiveness of different stimulus patterns on LTP induction in different layers of the EC. The results showed that TBS and SPWBS, compared with other stimulation patterns, induced larger degrees of LTP in the superficial (II-III) and deep 
A
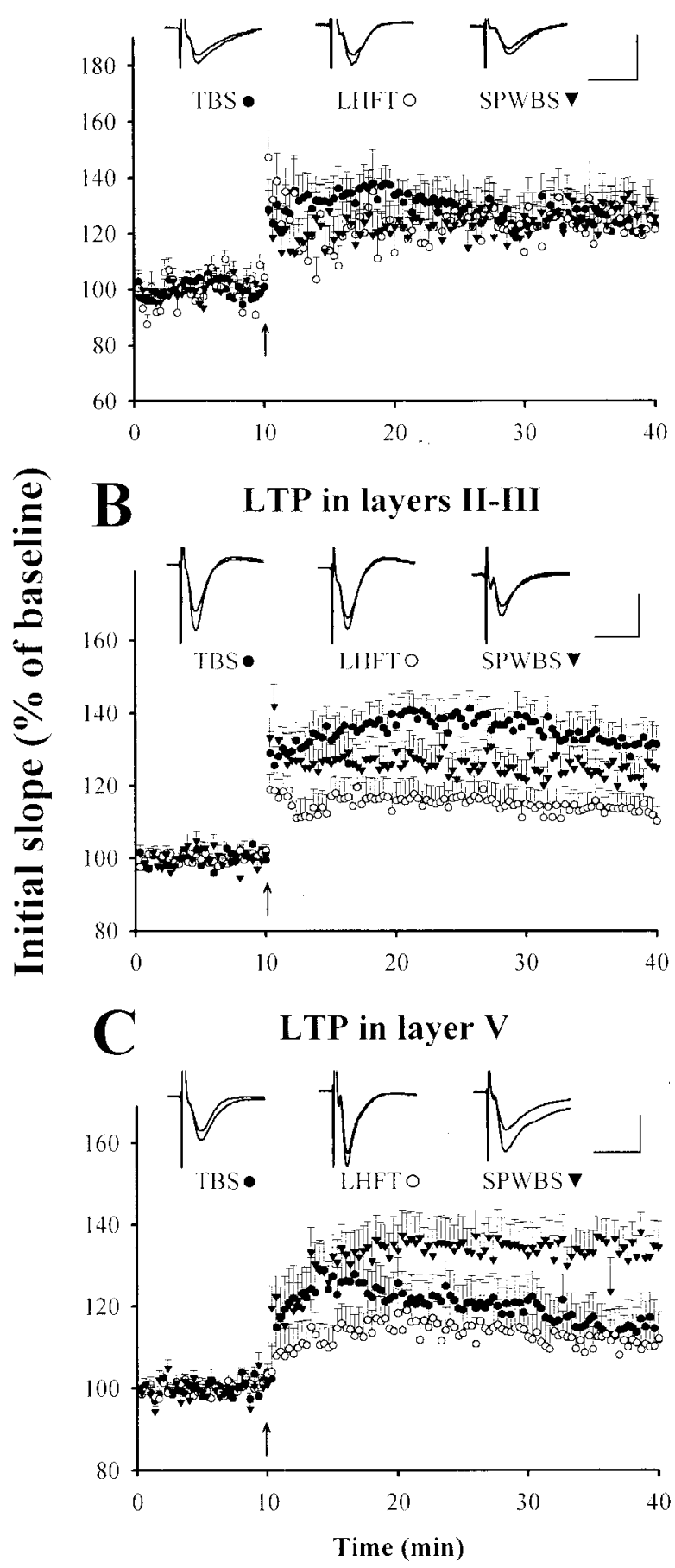

Figure 2. Induction of LTP by three different types of stimulation. Each scatterplot shows the average change in the initial slope of field EPSPs, expressed as a percentage of the baseline average after three types of burst stimulation ( $\bullet$ TBS; O, LHFT; $\boldsymbol{\nabla}$, SPWBS) in layers I, II-III, or V. $A$, LTP induction in layer I (LHFT, $n=10$; TBS, $n=10$; SPWBS, $n=$ 10). $B$, LTP induction in layers II-III (LHFT, $n=11$; TBS, $n=10$; SPWBS, $n=10$ ). $C$, LTP induction in layer V. (LHFT, $n=16$; TBS, $n=$ 19; SPWBS, $n=16$ ). Example field potential responses before and after LTP induction are shown superimposed on top. Each record is an average of five consecutive responses. The arrow indicates delivery of burst stimulation. Calibration: $10 \mathrm{msec}, 1 \mathrm{mV}$.

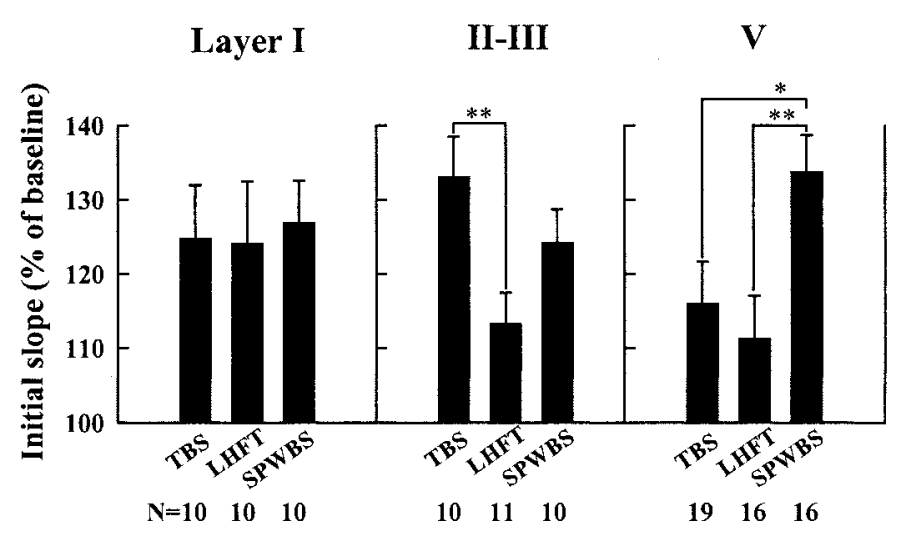

Figure 3. Average magnitudes of LTP induced by three different types of stimulation. The histograms show the average percentage changes in the initial slope of field EPSPs at 20-30 min after three different types of LTP-inducing stimulation in layers I, II-III, and V of the EC. Error bars indicate SEM; ${ }^{*} p<0.05 ;{ }^{* *} p<0.01$, respectively.

(V) layers of the EC, respectively (Figs. 2, 3). These results suggest that neural circuits in the superficial and deep layers of the EC may have evolved so that optimal induction of LTP is achieved during naturally occurring EEG that strongly modulates neuronal activities in each layer.

\section{Components of synaptic responses}

Because dendrites and associational axons of superficial EC neurons are primarily confined within the superficial EC, it is unlikely that the responses recorded in the deep EC involve superficial EC neurons. On the other hand, deep EC neurons extend their dendrites and send substantial associational projections to the superficial EC (Amaral and Witter, 1995; Dolorfo and Amaral, 1998). Thus, it is possible that both presynaptic and postsynaptic components of deep EC neurons are involved in the responses recorded in the superficial EC. Laminar profiles of field potential responses suggest, however, that the involvement is not very much. It was difficult to obtain sizable field potential responses (either positive or negative) by stimulating the superficial EC (layer I or II-III) and recording from the deep EC (testing postsynaptic elements of deep EC neurons in the superficial EC; data not shown) (Yun et al., 2000b, their Fig. 1). Likewise, it was difficult to obtain sizable negative field potential responses (current sink) by stimulating the deep EC (layer V or VI) and recording from the superficial EC (layer I or superficial layer III; testing presynaptic elements of deep EC neurons in the superficial EC; data not shown). Although results from slice preparations (400 $\mu \mathrm{m}$ thickness) should be interpreted cautiously, they nevertheless suggest that presynaptic or postsynaptic elements of deep EC neurons did not contribute much to the responses recorded in the superficial EC in the present study. It would be interesting to find out optimal stimulus patterns for inducing LTP in synapses made by the presynaptic or postsynaptic elements of deep EC neurons in the superficial EC in future studies.

Regarding layer I versus layers II-III responses, they probably share postsynaptic elements, because layer I is relatively devoid of neurons, and layers II-III neurons extend their dendrites to layer I (Amaral and Witter, 1995). Laminar profiles of field potential responses suggest, however, that primarily different sets of synapses were activated by layer I versus layers II-III stimulation. For example, stimulation of layers II-III evoked positive and negative field potential responses in layer I and superficial layer 
III, respectively (Yun et al., 2000b, their Fig. 1). Anatomical data also indicate large variations in the distribution of afferent and intrinsic associational fibers between layer I and other layers (Swanson et al., 1987; Witter, 1993; Dolorfo and Amaral, 1998).

\section{Cellular mechanisms underlying variation in effective stimulus patterns}

Why are TBS and SPWBS more effective in eliciting LTP in layers II-III and V of the EC, respectively? In the hippocampus, theta $(\sim 5 \mathrm{~Hz})$ is the optimal frequency for the induction of LTP (Larson et al., 1986). GABA A receptor-mediated fast IPSPs normally exert powerful influences on hippocampal neurons, preventing strong depolarization of postsynaptic neurons in response to a short burst. Priming stimulation temporarily suppresses fast IPSPs so that theta frequency burst stimulation induces maximal depolarization, which in turn leads to maximal activation of NMDA receptor-mediated currents (Larson and Lynch, 1986, 1988; Mott and Lewis, 1991). It is possible that such mechanisms operate in layers II-III, but are less prominent in layer V of the EC. Consistent with this possibility, anatomical (Kohler et al., 1985; Wouterlood et al., 1995) and physiological (Jones, 1993; Funahashi and Stewart, 1998) studies have indicated that inhibitory influences are much stronger in the superficial than deep EC. Moreover, during repetitive stimulation, both fast and slow IPSPs of superficial EC neurons are suppressed (Funahashi and Stewart, 1998). These features (strong IPSPs and their suppression by priming stimulation) in the superficial EC could explain why TBS induced maximal LTP in layers II-III.

When compared with the superficial EC, the influence of IPSPs is less prominent in the deep EC (Jones, 1993; Funahashi and Stewart, 1998). Under the circumstance of weak inhibitory control, prolonged stimulation (more pulses in a burst) would lead to larger postsynaptic depolarization and hence more activation of NMDA currents. SPWBS and TBS delivered 20 and 4 pulses in a burst, respectively. This may explain why SPWBS was more effective than TBS in inducing LTP. Then a question arises regarding why SPWBS induced larger LTP than LHFT, which is the longest stimulus train (1 sec). Funahashi and Stewart (1998) have shown that IPSPs are not completely suppressed but rapidly reach a plateau level and that repetitive stimulation at $50 \mathrm{~Hz}$ results in progressive summation of IPSPs in superficial and deep EC neurons. It is then likely that only the initial part of LHFT is effective in activating postsynaptic neurons. The later part of the LHFT may not induce sufficient postsynaptic depolarization because of summated IPSPs. In such a case, single prolonged stimulation would be less effective than multiple short bursts in inducing LTP. In addition, progressive desensitization of NMDA receptors may have contributed to ineffectiveness of LTP induction by prolonged stimulation. NMDA receptors are known to desensitize with slower time courses than AMPA receptors (Zorumski and Thio, 1992). Combined, it is possible that multiple short bursts with the sufficient number of pulses $(>4)$ in each burst (such as SPWBS) are the most effective stimulus pattern in the EC layer $\mathrm{V}$.

\section{Learning mode versus replay mode}

Because theta frequency EEG is preferentially observed when an animal is engaged in exploratory behaviors, whereas SPWs are observed during consummatory behaviors and slow-wave sleep, a hypothesis has been proposed that theta rhythm and SPWs represent learning and replay mode of the hippocampal formation, respectively (Buzsaki, 1989). Combined with anatomical and physiological studies that indicate functional segregation between superficial and deep layers of the EC (see introductory remarks), these findings suggest that incoming sensory information from the neocortex is relayed via the superficial EC to the hippocampus when an animal is learning new information, and the mode of operation during this phase is theta frequency oscillation. On the other hand, in decoding (replay) phase, memories stored in the hippocampus are reactivated and propagated to the neocortex via the deep EC. The operational mode in this phase is SPW oscillation (Buzsaki, 1989, 1996; Chrobak et al., 2000). In the present study we found that rhythmic stimulation patterned after theta and SPW EEG induced the largest degrees of LTP in the superficial (II-III) and deep (V) layers of the EC, respectively. It is then likely that synaptic weight enhancement occurs preferentially in the superficial EC during the learning mode, whereas the deep EC is the major locus of synaptic weight change during the replay mode.

The present results by no means indicate that synaptic potentiation occurs only in the superficial layers during the theta phase and only in the deep layers during the SPW phase. In the present study, SPWBS induced significant, albeit smaller, LTP in layers II-III, and TBS induced significant LTP in layer V. TBS (at $7 \mathrm{~Hz}$ ) applied to CA1-subiculum also induced LTP in the deep EC and perirhinal cortex in vivo (Otto and Eichenbaum, 1993; Cousens and Otto, 1998). These results suggest that LTP can occur in both the superficial and deep EC during both theta (learning mode) and SPW phase (replay mode), but the magnitudes are different. A single-unit recording study in behaving animals has shown interesting coding properties of deep EC neurons during the theta phase, suggesting that EC deep layers transmit information back to the neocortex during the theta phase (Frank et al., 2000). LTP in the deep layers during the theta phase may function as a mechanism that associates sensory activation patterns in the neocortex and hippocampal memories. This would enable reactivation of original sensory activation patterns in the neocortex based on hippocampus-initiated neuronal activities. It may therefore function as a means to link multiple sensory activation patterns in different regions of the neocortex (Teyler and DiScenna, 1986). Currently the extent to which LTP is induced in layer $\mathrm{V}$ during the theta phase in vivo is unknown as are the properties of information transmission and induction of LTP in superficial layers during the SPW phase in vivo. These are crucial information in formulating hippocampal-neocortical interactions in encoding and retrieval of memories. Future studies in behaving animals are required to obtain such information.

\section{LTP in layer I}

It is not clear why the three different stimulus patterns, unlike in the other layers, induced similar degrees of LTP in layer I. That effect may be attributable to dependence of LTP induction on voltage-dependent calcium channels (VDCCs) in layer I. Whereas LTP in layers II-III and V was dependent only on activation of NMDA receptors, LTP in layer I was completely blocked only after blocking both NMDA receptors and VDCCs (data not shown). Although the three different stimulus patterns induced different amounts of depolarization and calcium influx through NMDA receptors, additional depolarization and calcium influx through VDCCs may have made overall calcium influx similar across the three stimulus patterns. In other words, the nonlinearity offered by VDCCs may have hidden differences in effectiveness of the three stimulus patterns in inducing calcium influx. Similarly, nonlinear amplification of backpropagating ac- 
tion potentials in the distal dendrites may have contributed to this observation. Backpropagating action potentials are amplified three to four times in the distal, but not in proximal dendrites of layer $\mathrm{V}$ pyramidal cells in somatosensory cortical slices (Stuart and Hausser, 2001). If EC pyramidal neurons operate in a similar way, amplification of backpropagating action potentials in the distal dendrites will confer nonlinearity in dendritic depolarization. Finally, it is possible that types of synapses are different across different EC layers.

\section{REFERENCES}

Alonso A, de Curtis M, Llinas R (1990) Postsynaptic Hebbian and non-Hebbian long-term potentiation of synaptic efficacy in the entorhinal cortex in slices and in the isolated adult guinea pig brain. Proc Natl Acad Sci USA 87:9280-9284.

Amaral DG, Witter MP (1995) Hippocampal formation. In: The rat nervous system (Paxinos G, ed), pp 443-493. San Diego: Academic.

Buzsaki G (1989) Two-stage model of memory trace formation: a role for "noisy" brain states. Neuroscience 31:551-570.

Buzsaki G (1996) The hippocampo-neocortical dialogue. Cereb Cortex 6:81-92.

Buzsaki G, Leung LS, Vanderwolf CH (1983) Cellular basis of hippocampal EEG in the behaving rat. Brain Res 6:139-171.

Cheong MY, Yun SH, Mook-Jung I, Joo I, Huh K, Jung MW (2001) Cholinergic modulation of synaptic physiology in deep layer entorhinal cortex of the rat. J Neurosci Res 66:117-121.

Chrobak JJ, Buzsaki G (1994) Selective activation of deep layer (V/VI) retrohippocampal cortical neurons during hippocampal sharp waves in the behaving rat. J Neurosci 14:6160-6170.

Chrobak JJ, Lorincz A, Buzsaki G (2000) Physiological patterns in the hippocampal-entorhinal cortex system. Hippocampus 10:457-465.

Cousens G, Otto TA (1998) Induction and transient suppression of longterm potentiation in the peri- and postrhinal cortices following thetarelated stimulation of hippocampal field CA1. Brain Res 780:95-101.

Dolorfo CL, Amaral DG (1998) Entorhinal cortex of the rat: organization of intrinsic connections. J Comp Neurol 398:49-82.

Frank LM, Brown EN, Wilson MA (2000) Trajectory encoding in the hippocampus and entorhinal cortex. Neuron 27:169-178.

Frank LM, Brown EN, Wilson MA (2001) A comparison of the firing properties of putative excitatory and inhibitory neurons from CA1 and the entorhinal cortex of the awake behaving rat. J Neurophysiol 86:2029-2040.

Funahashi M, Stewart M (1998) GABA receptor-mediated post-synaptic potentials in the retrohippocampal cortices: regional, laminar and cellular comparisons. Brain Res 787:19-33.
Jones RS (1993) Entorhinal-hippocampal connections: a speculative view of their function. Trends Neurosci 16:58-64.

Kohler C, Wu JY, Chan-Palay V (1985) Neurons and terminals in the retrohippocampal region in the rat's brain identified by anti-gammaaminobutyric acid and anti-glutamic acid decarboxylase immunocytochemistry. Anat Embryol 173:35-44.

Larson J, Lynch G (1986) Induction of synaptic potentiation in hippocampus by patterned stimulation involves two events. Science 232:985-988.

Larson J, Lynch G (1988) Role of $N$-methyl-D-aspartate receptors in the induction of synaptic potentiation by burst stimulation patterned after the hippocampal theta-rhythm. Brain Res 441:111-118.

Larson J, Wong D, Lynch G (1986) Patterned stimulation at theta frequency is optimal for the induction of hippocampal long-term potentiation. Brain Res 368:347-350.

Mott DD, Lewis DV (1991) Facilitation of the induction of long-term potentiation by GABAB receptors. Science 252:1718-1720.

Otto T, Eichenbaum H (1993) Long-term potentiation in entorhinal cortex induced by theta-pattern stimulation of CA1. Soc Neurosci Abstr 19:906.

Stuart GJ, Hausser M (2001) Dendritic coincidence detection of EPSPs and action potentials. Nat Neurosci 4:63-71.

Swanson LW, Köhler C, Björklund A (1987) The limbic region. I. The septohippocampal system. In: Handbook of chemical neuroanatomy. Integrated Systems of the CNS (Björklund A, Hökfelt T, Swanson LW, eds) Vol 5, pp 125-277. Amsterdam: Elsevier.

Teyler TJ, DiScenna P (1986) The hippocampal memory indexing theory. Behav Neurosci 100:147-154.

Witter MP (1986) A survey of the anatomy of the hippocampal formation, with emphasis on the septotemporal organization of its intrinsic and extrinsic connections. In: Excitatory amino acids and epilepsy (Schwarcz R, Ben-Ari Y, eds), pp67-82. New York: Plenum.

Witter MP (1993) Organization of the entorhinal-hippocampal system: a review of current anatomical data. Hippocampus 3:33-44.

Wouterlood FG, Hartig W, Bruckner G, Witter MP (1995) Parvalbumin-immunoreactive neurons in the entorhinal cortex of the rat: localization, morphology, connectivity and ultrastructure. J Neurocytol 24:135-153.

Yeckel MF, Berger TW (1998) Spatial distribution of potentiated synapses in hippocampus: dependence on cellular mechanisms and network properties. J Neurosci 18:438-450.

Yun SH, Cheong MY, Mook-Jung I, Huh K, Lee C-J, Jung MW (2000a) Cholinergic modulation of synaptic transmission and plasticity in entorhinal cortex and hippocampus of the rat. Neuroscience 97:671-676.

Yun SH, Huh K, Jung MW (2000b) Selective enhancement of nonNMDA receptor-mediated responses following induction of long-term potentiation in entorhinal cortex. Synapse 35:1-7.

Zorumski CF, Thio LL (1992) Properties of vertebrate glutamate receptors: calcium mobilization and desensitization. Prog Neurobiol 39:295336. 